\title{
Early post-myocardial infarction survival in MRL mice is mediated by attenuated apoptosis and inflammation but depends on genetic background
}

\author{
Darlene L. Hunt ${ }^{1}$, Patrick H. CampbelI ${ }^{1}$, Alexander C. Zambon ${ }^{2,8}$, Karen Vranizan ${ }^{3,4}$, Sylvia \\ M. Evans ${ }^{5}$, Hai-Chien Kuo ${ }^{6}$, Ken D. Yamaguchi ${ }^{7}$, Jeffrey H. Omens ${ }^{1,8}$, and Andrew D. \\ McCulloch ${ }^{1}$ \\ ${ }^{1}$ Department of Bioengineering, University of California at San Diego, La Jolla, CA \\ ${ }^{2}$ Department of Pharmacology, University of California at San Diego, La Jolla, CA \\ ${ }^{3}$ Gladstone Institute of Cardiovascular Disease, San Francisco, CA \\ ${ }^{4}$ Functional Genomics Laboratory, University of California, Berkeley, CA \\ ${ }^{5}$ School of Pharmacy, University of California at San Diego, La Jolla, CA \\ ${ }^{6}$ Department of Cardiovascular Research, Berlex Biosciences, Richmond, CA \\ ${ }^{7}$ Department of Computational Biology, Berlex Biosciences, Richmond, CA \\ ${ }^{8}$ Department of Medicine, University of California at San Diego, La Jolla, CA
}

\begin{abstract}
The Murphy Roths Large (MRL) mouse, a strain capable of regenerating right ventricular myocardium, has a high post-myocardial infarction (MI) survival rate compared with C57BL/6J (C57) mice. The biological processes responsible for this survival advantage are unknown. To assess the effect of genetic background, the LG/J strain, which harbors $75 \%$ of the MRL composite genome, was included in the study. The MRL survival advantage versus C57 mice ( $92 \%$ vs. $68 \%, P<0.05$ ) occurred primarily in the first 5 days; $\mathrm{LG} / \mathrm{J}$ survival was intermediate $(P$ $=$ NS $)$. Microarray data analysis revealed an attenuation of apoptotic $(P<0.05)$ and stress response transcripts in MRL hearts compared with C57 hearts after MI. Supporting the microarray results, there were fewer TUNEL-positive cells 1 day post-MI in MRL infarcts compared with C57 infarcts $(P=0.001)$ and fewer CD45-positive cells in the MRL infarct border zone 2 days post-MI $(P<0.01)$. LG/J results were intermediate $(P=\mathrm{NS})$. MRL hearts had smaller infarct scars and attenuated ventricular dilation 30 days post-MI compared with $\mathrm{C} 57$ hearts $(P<0.05)$. We conclude that the early post-MI survival advantage of MRL mice over the C57 strain is mediated at least in part by reductions in apoptosis and inflammatory infiltration, and that these reductions may influence chronic remodeling. The intermediate survival, apoptosis and inflammation profile of LG/J mice suggests this high tolerance for MI in the MRL could be derived from its shared genetic background with the LG/J.
\end{abstract}

Address correspondence to: Andrew D. McCulloch, PhD, Room 231 Powell-Focht Bioengineering Hall, 9500 Gilman Drive, La Jolla, CA 92093-0412, amcculloch@ ucsd.edu Telephone: (858) 534-2547 Fax: (858) 332-1706.

Note: The corresponding author will sign any required forms in place of Karen Vranizan, deceased June 8, 2009.

Disclosures

Berlex Biosciences provided financial support by purchasing the Affymetrix microarrays used in this study. 


\section{Keywords}

Heart; inflammation; ischaemia

\section{Introduction}

Heart disease remains the leading cause of death in the industrialized world, with myocardial infarction (MI) as one of its major causes. Because of the limited self-renewal capability of cardiac myocytes, wound repair consists largely of replacing necrotic myocardium with collagenous scar tissue (Pfeffer et al., 1979; Pfeffer \& Braunwald, 1990). Early complications can include pump failure and myocardial rupture, the latter accounting for up to $10 \%$ of acute mortality (van den Borne et al., 2009). Chronically, large infarcts cause progressive ventricular dilation (Pfeffer et al., 1991), leading the heart into a "vicious cycle" of decompensation and eventual failure.

The MRL mouse has emerged as an intriguing model to study regenerative cardiac healing. Bred as a wildtype control for the lymphoproliferative MRL-Fas ${ }^{p r}$, the MRL/MpJ was generated through a series of crosses with LG/J (75\%), AKR/J (12.6\%), C3H/HeDi (12.1\%), and C57BL/6J (0.3\%). Its potential for regenerative healing was discovered when ear hole punches used for colony marking healed rapidly and closed fully within 30 days, with complete replacement of normal tissue architecture (Clark et al., 1998). The LG/J shares this fast-healing trait (Li et al., 2001; Blankenhorn et al., 2009), though ear punch wounds of male mice do not close completely (Blankenhorn et al., 2009).

Leferovich and colleagues (2001) were the first to report that MRL mice are capable of regenerating right ventricular (RV) myocardium after cryoprobe injury. In contrast to RV cryoinjury, large-scale regeneration of left ventricular (LV) myocardium was not observed after ischemia-reperfusion (Abdullah et al., 2005) or permanent coronary ligation (Oh et al., 2004; Naseem et al., 2007; Cimini et al., 2008; Grisel et al., 2008; Robey \& Murry, 2008). However, one group (Naseem et al., 2007) reported the presence of myocytes undergoing cell division in the infarct border zone, as well as significantly increased chronic survival compared with C57BL/6J (C57) controls. The reasons for these differences are unknown.

The purpose of the present study was to elucidate the biological processes responsible for increased post-MI survival in the MRL, and to investigate whether its major parental strain, the LG/J, also displays heightened survival and healing traits. Here we show that increased acute survival in MRL mice post-infarct results from the absence of ventricular rupture, and may be a consequence of decreased apoptosis and inflammatory infiltration compared to the C57. The LG/J is also susceptible to rupture but has survival, apoptosis, and inflammation characteristics intermediate between the C57 and the MRL. Chronically, MRL hearts heal with reduced infarct scar size and indices of LV dilation and infarct expansion compared with C57 hearts.

\section{Materials and Methods}

\section{Ethical approval}

Male C57BL/6J, MRL/MpJ, and LG/J mice were obtained from the Jackson Laboratories and housed in a vivarium according to institutional guidelines. Animals were between 15 and 21 weeks old at the time of surgery. All animal studies and husbandry were conducted under approved University of California, San Diego Animal Subjects protocols in AAALAC-approved facilities. 


\section{Myocardial infarction surgery}

Coronary artery ligation surgery was performed as described previously (Campbell et al., 2008). Briefly, animals were intubated, mechanically ventilated, and left thoracotomy was performed under inhalant isoflurane anesthesia (induced at 5\% and maintained at $2 \%$ in $100 \%$ oxygen $)$. Buprenorphine $(0.1 \mathrm{mg} / \mathrm{kg})$ was given subcutaneously after intubation as a postoperative analgesic. The left coronary artery was ligated with a 7-0 silk suture. The appearance of paleness distal to the suture confirmed ischemia. Animals were rehydrated with warm saline, removed from the ventilator, and allowed to recover on a watercirculating heating pad with access to oxygen. After regaining consciousness, mice were transferred to a clean cage and monitored for signs of distress. An additional dose of buprenorphine was given the following day if indicated.

\section{Survival studies}

The MI procedure described above was performed on C57, MRL, and LG/J mice. Subsets of all three strains that were sacrificed after 1,2,3,5, or 30 days became censored subjects in a Kaplan-Meier survival analysis. Deaths were recorded on a daily basis. Hearts of animals surviving the MI procedure were excluded from the study if the ligature was outside a predetermined tolerance distance from the left atrium when MI was present, or if visual inspection revealed only trace ischemia, indicating unsuccessful ligation. Therefore, the number of animals entered into the Kaplan-Meier analysis is smaller than the number that survived the procedure.

\section{Initial infarct size}

Animals were anesthetized with isoflurane, and maintained at 5\% to ensure deep anesthesia. A thoracotomy was performed, the heart was removed, and immersed in an ice-cold, hyperkalemic Krebs-Henseleit buffer containing 2,3-butanedione monoxime (BDM, Sigma). To delineate the area at risk, the heart was retrograde perfused with $1 \%$ Evans Blue dye. LVs were cut into $1 \mathrm{~mm}$ slices and incubated in buffered 2,3,5-triphenyl tetrazolium chloride (TTC, Sigma) at $37^{\circ} \mathrm{C}$ for 20 minutes, then fixed overnight in $10 \%$ zinc formalin (Electron Microscopy Sciences) or 4\% paraformaldehyde. Infarct size was expressed as the ratio of total infarct area (pale color) to total tissue area. For hearts also stained with Evans Blue, infarct size was normalized to the area at risk, defined as the sum of the infarct area and the area of myocardium that stained red but not blue.

\section{Hemodynamic measurements}

One day after the MI procedure, animals were anesthetized intraperitoneally with a mixture of ketamine $(100 \mathrm{mg} / \mathrm{kg})$ and xylazine $(10 \mathrm{mg} / \mathrm{kg})$, intubated, and mechanically ventilated with $100 \%$ oxygen as described above. Depth of anesthesia was determined every 30 minutes using a toe pinch stimulus, and a maintenance dose was given if needed. A 1.4F MikroTip catheter (Millar Instruments) was advanced into the LV through the right carotid artery. Invasive baseline hemodynamic measurements were obtained using the catheter connected to a control unit (Millar Instruments) and a data acquisition board (National Instruments). Hemodynamic parameters were calculated with PVAN software (Millar Instruments).

\section{Histology and immunohistochemistry}

Hearts were harvested as described above, and the ventricles were either fixed in $10 \%$ zinc formalin for 24 hours and embedded in paraffin, or fresh frozen in Optimal Cutting Temperature (OCT) compound (Tissue-Tek). For hearts isolated on day 2 post-MI, $10-\mu \mathrm{m}$ sections were obtained at the midventricular level and stained for CD45 (BD Biosciences primary antibody) (Heymans et al., 1999). Nuclei were counterstained with hematoxylin. 6- 
7 non-overlapping images covering both infarct border regions for each heart were obtained (AxioCam HRc with AxioVision software). Blinded observers counted the number of $\mathrm{CD} 45^{+}$cells in each image. Data are reported as the number of $\mathrm{CD}^{+} 5^{+}$cells per $\mathrm{mm}^{2}$ of tissue.

\section{Gelatin Zymography}

Samples were homogenized in $10 \mathrm{mM}$ HEPES, pH 7.5, $150 \mathrm{mM} \mathrm{NaCl}, 0.2 \mathrm{mM}$ EDTA, 25\% glycerol, with protease inhibitor cocktail (Sigma). Samples (10 $\mathrm{mg}$ of protein) were loaded onto a $10 \%$ SDS-PAGE gel substituted with $0.1 \%$ gelatin and then stained with $0.5 \%$ coomassie blue. MMP-9 protein standards (Chemicon) were loaded on all gels. Bands of MMP-9 gelatinolytic activity were digitally quantified with Image $\mathrm{J}$ and normalized to standards.

\section{Infarct size quantification and morphometric measurements}

For hearts isolated on day 5 or day 30 post-MI, 8-10 evenly spaced sections from apex to base were stained for infarct size measurement. Day 5 post-MI hearts were stained with H\&E or Masson's trichrome, and day 30 post-MI hearts were stained with Masson's trichrome. Infarct size was determined using an endocardial perimeter measurement method modified from Pfeffer et al. (1979). LV dilation and infarct expansion indices (Boyle \& Weisman, 1993; Reffelmann et al., 2003) were calculated for the day 30 post-MI hearts. Infarct thickness was measured in stained sections for day 5 and day 30 post-MI hearts.

\section{Apoptosis detection and quantification by TUNEL assay}

Twenty-four hours after MI, mice were sacrificed and the hearts were frozen in OCT as described above. Apoptosis detection was performed by a Terminal deoxynucleotidyl Transferase (TdT) dUTP Nick End Labeling (TUNEL) assay (CardioTACS In Situ Apoptosis Detection Kit, R\&D Systems). Approximately 20 randomly chosen, nonoverlapping images covering the infarct and border regions were acquired using a $\times 40$ objective. Blinded observers counted the number of TUNEL-positive nuclei and TUNELnegative nuclei in each image. Only those TUNEL-positive nuclei closely associated with myocytes were counted. An apoptotic index, expressed as percent TUNEL-positive nuclei to total nuclei, was calculated for each heart.

\section{Gene expression analysis using MOE430A microarrays}

Animals were anesthetized with isoflurane as described above, and a thoracotomy was performed. The heart was removed and arrested, and the right ventricle was quickly dissected away. LV infarct (including the border zone) and non-infarct areas were carefully separated. Tissue pieces were separately snap-frozen in liquid nitrogen and stored at $-80^{\circ} \mathrm{C}$. Total RNA was isolated by Berlex Biosciences (Richmond, CA; now Bayer HealthCare Pharmaceuticals) and shipped to Expression Analysis, Inc. (Durham, NC) for hybridization to Affymetrix MOE430A microarrays.

\section{Comparisons, statistical analysis and HOPACH clustering}

Affymetrix CEL files were quality controlled with R (www.r-project.org) packages available from the Bioconductor (Gentleman et al., 2004) website (www.bioconductor.org). Two arrays were excluded due to hybridization anomalies. Expression data are available at the Gene Expression Omnibus (www.ncbi.nlm.nih.gov/geo/) under the accession number GSE19322. The gcRMA package (Wu \& Irizarry, 2004) was used to generate $\log _{2}$ expression signal values. Two separate sets of moderated $F$-statistics (Smyth, 2004) were generated with the limma package to determine if genes were differentially expressed (i.e., had significant interaction $P$ values) between C57 and MRL samples in the infarct or the 
non-infarct regions across the time course $(0,1$, and 5 days post-MI). Limma uses linear models to analyze expression datasets (Smyth, 2004). The data set was filtered for interaction $P<0.05$ in either the infarct or the non-infarct regions (1005 probe sets) and at least a 50\% fold change in any comparison of baseline to post-infarct time for each genotype (MRL, C57) for each region (infarct, non-infarct), including baseline expression differences between the two mouse lines ( 739 probe sets). These were filtered to remove duplicate gene symbols (688 probe sets), and were then clustered using the HOPACH clustering algorithm (van der Laan \& Pollard, 2001).

\section{MAPPFinder results}

Second-level clusters obtained using the HOPACH algorithm were annotated with Gene Ontology (GO) terms (www.geneontology.org) using the MAPPFinder program (Doniger et $a l ., 2003)$. These results were filtered to include terms with at least 3 changed genes and a $Z$ score of 2 or greater (approximately equivalent to $P<0.05$ ). Groups of parent-child relationships were identified and reduced so that only the term with the highest $\mathrm{Z}$ score is reported.

\section{Real-time quantitative RT-PCR}

Isolated hearts with and without MI were separated as described above, and infarct and noninfarct areas were stored separately in RNALater (Qiagen). Total RNA was isolated using the RNeasy Midi kit (Qiagen). cDNA was synthesized from 100 ng of RNA using SuperScript II reverse transcriptase (Invitrogen). Oligo(dT) 12-18 $_{18}$ was used as a primer. Realtime quantitative reverse transcriptase polymerase chain reaction (qRT-PCR) was performed (Applied Biosystems 7300) using 90 ng of cDNA per reaction. Glyceraldehyde 3-phosphate dehydrogenase (GAPDH) was used as an internal control. Fold induction or repression was calculated relative to uninjured (day 0 ) controls of the same strain and region after adjusting for GAPDH expression, using the comparative $\mathrm{Ct}(\Delta \Delta \mathrm{Ct}$ ) method (Ashton \& Headrick, 2007).

\section{Statistics}

Data were analyzed using GraphPad Prism 5 software. Survival curves were generated according to the Kaplan-Meier method, and a logrank test was used to check for differences between strains. To account for animals that were sacrificed on or before 5 days post-MI, 5day rupture rates for all 3 strains were calculated using a separate Kaplan-Meier analysis, where "death" events were rupture deaths only. Data including 3 or more groups were analyzed by the Kruskal-Wallis statistic and Dunn post-hoc tests or two-way ANOVA and Bonferroni post-hoc tests. Two-group analysis was performed using an unpaired, two-tailed t-test. Surgical outcomes data were analyzed using a Chi square test. The significance level was set at $\alpha=0.05$. All data are reported as mean \pm S.E.M.

\section{Results}

\section{MRL mice have a survival advantage compared to C57 mice}

A Kaplan-Meier survival analysis of all 3 strains showed that MRL mice had a marked survival advantage over C57 mice, and that most of this difference occurred in the first 5 days post-MI (Fig. 1). LG/J survival also dropped after 5 days, to a rate between the C57 and MRL strains, but the difference was not statistically significant. The cause of death in 17 of the $21 \mathrm{C} 57$ and both of the $2 \mathrm{LG} / \mathrm{J}$ mice that died during this period (see Table 1) was left ventricular rupture, determined by the presence of blood in the chest cavity at necropsy. The 5 MRL animals that died in the first 5 days appeared to succumb to pulmonary edema, diagnosed by the presence of fluid in the lungs. The MRL rupture rate of $0 \%$ was 
significantly different from that of C57 and LG/J mice (Table 1 and Supplemental Figure S1). One C57 animal died of rupture outside of the 5-day period; there were no other rupture deaths (Supplemental Figure S1). Difficulty visualizing the target artery in LG/J hearts led to a lower MI success rate than the other strains.

These differences in survival could not be attributed to inconsistent initial infarct sizes or hemodynamic parameters. Infarct size 1 day after surgery, determined by TTC staining, was not statistically different between the strains (Fig. 2A). Owing to the low sample number in the LG/J group, this conclusion cannot be supported with high power. This lack of difference held when infarct size was normalized to the area at risk (C57 $n=6$, MRL $n=7$, $\mathrm{LG} / \mathrm{J} n=3, P=0.70$; data not shown). Analysis of hemodynamic data (Table 2) did not indicate that MRL mice had a more favorable hemodynamic environment than C57 or LG/J mice 1 day post-MI.

In a cohort of animals, we measured MMP-9 activity on day 3 post-MI to determine if increased activity could be a factor in the high rupture rate of C57 mice compared with the other strains. Gelatin zymography for MMP-9 in all three strains revealed a slight trend toward reduced gelatinolytic activity in the MRL LV and RV compared to the C57 and LG/J LV and RV, but the trend did not reach statistical significance (Supplemental Figure S2).

In another cohort of animals, we measured infarct size and thickness on day 5 post-MI to determine if increased survival in the MRL strain could be related to attenuated infarct expansion (Hutchins \& Bulkley, 1978) or preserved thickness compared to C57 or LG/J hearts (Fig. 2B, C). There were no significant differences in infarct size between groups (Fig. 2B). There were also no significant differences in infarct thickness between the groups (Fig. 2C). Based on these results, the survival advantage in MRL mice could not be attributed to attenuated infarct expansion or preserved infarct thickness.

\section{Enhanced reparative response in MRL infarcts}

To generate hypotheses regarding which biological processes could contribute to the increased survival of MRL mice, gene expression was analyzed before (day 0) and 1 and 5 days after MI in the infarct and non-infarct regions of C57 and MRL hearts ( $n=4$ per group except C7 day $0 n=3$ ). HOPACH clustering (van der Laan \& Pollard, 2001) of the 688 filtered transcripts identified the major differences in patterns of gene expression (Fig. 3).

Using MAPPFinder (Doniger et al., 2003), clusters were annotated with Gene Ontology (www.geneontology.org) terms (Fig. 3) to understand the functional significance of the changed genes. Transcripts related to known effects of MI injury, such as response to stress and inflammation, appeared in the cluster with progressive induction of expression in the MRL (PI-MRL) and the cluster with early induction of expression in the C57 and MRL (EIC57/MRL; Fig. 3). However, there was also clear differential regulation. The EI-C57/MRL cluster contained transcripts associated with the induction of apoptosis and negative regulation of cell proliferation that were not present in the PI-MRL cluster. Apoptotic transcripts were also present in the PI-C57 cluster. In contrast, the PI-MRL cluster contained numerous transcripts related to reparative processes, including those associated with the GO terms, "positive regulation of development" and "cell motility." Additional terms described cellular constituents such as "extracellular matrix," and biological processes such as "angiogenesis."

To validate this analysis, three transcripts were chosen for quantification with real-time PCR (Fig. 4). Platelet-derived growth factor beta (Pdgfb), a cell survival factor (Au et al., 2005), and chondroitin sulfate proteoglycan 4 (Cspg4), a mediator of cell adhesion and proliferation (Ugur et al., 2001), were members of the PI-MRL cluster. Fas death domain-associated 
protein (Daxx), a pro-apoptotic factor (Yang et al., 1997), was a member of the PI-C57 cluster. The expression patterns of these genes using qRT-PCR (Fig. 4B) were similar to the patterns in the microarray data (Fig. 4A).

In summary, MRL and C57 hearts both appear to express transcripts associated with a characteristic response to MI injury, but differential regulation is also apparent. MRL hearts may express more transcripts associated with reparative processes, while C57 hearts may express more transcripts associated with deleterious processes such as apoptotic cell death.

\section{MRL infarct tissue is less susceptible to early cell death}

To test the hypothesis suggested by the microarray analysis that differential regulation of apoptosis could be a significant factor in the increased survival of MRL mice post-MI, a TUNEL assay was performed 1 day after coronary ligation in hearts from each strain (Fig. $5 \mathrm{~A}, \mathrm{~B}$ ). There was a 6-fold decrease in the number of TUNEL-positive cells closely associated with myocytes in the MRL infarct region compared to the C57 infarct (Fig. 5D). The LG/J apoptotic index was intermediate ( $P=\mathrm{NS}$; Fig. 5D). TUNEL-positive cells were not observed in remote LV myocardium or the interventricular septum (Fig. 5C), or in uninjured controls (not shown).

\section{MRL hearts have attenuated CD45 ${ }^{+}$cell infiltration}

Microarray analysis results suggested that the MRL inflammatory response could be delayed compared to the C57, due to the progressive pattern of induction in the PI-MRL cluster compared to the EI-C57/MRL cluster. Leukocyte infiltration was investigated on day 2 postMI by immunohistochemical staining for CD45 (Fig. 6 A, B), which labels all leukocytes (Pattanapanyasat et al., 1994). There was a 5-fold decrease in the number of $\mathrm{CD}^{4} 5^{+}$cells in the MRL infarct border zone compared to the C57 border zone (Fig. 6D), consistent with an attenuated inflammatory response. The LG/J response was intermediate $(P=\mathrm{NS} ;$ Fig. 6D).

\section{MRL hearts have smaller infarct scars and attenuated remodeling}

To determine if there was a different chronic response to MI injury between the strains, subsets of MRL and C57 mice were sacrificed after 30 days ( $n=11$ and 10, respectively) and hearts were examined histologically. Mature MRL infarct scars were significantly smaller than C57 scars (Fig. 7A, B). Indices of LV dilation (Fig. 7C) and infarct expansion (Fig. 7D) were also attenuated. There was a trend toward increased scar thickness in MRL hearts, but the difference did not reach statistical significance ( $P=0.08$, data not shown).

\section{Discussion}

The results presented above show that increased survival in MRL mice compared with C57 mice is attributable to the absence of ventricular rupture in the acute phase post-MI, and provide the first data on post-MI survival, apoptosis, and inflammation in its parental strain, the LG/J. Lack of rupture in the MRL could not be attributed to smaller infarct size, more favorable hemodynamics, or attenuated infarct expansion. We performed a microarray study to generate hypotheses that could potentially explain the survival advantage of the MRL over the C57, and our analysis suggested that even in the first $1-5$ days after MI, MRL hearts began to express transcripts associated with wound repair while C57 hearts expressed transcripts associated with cell death. These results were confirmed with tissue-level assays that showed significantly fewer TUNEL-positive cells and less $\mathrm{CD} 45^{+}$cell infiltration in the MRL infarct, parameters that have not previously been investigated after acute coronary artery ligation in the MRL. Similar to the survival results, TUNEL-positive cells and CD45 ${ }^{+}$ cell infiltration in LG/J hearts were intermediate between the C57 and MRL strains. Thirty 
days post-MI, MRL infarct scars were smaller than C57 scars, which could not be attributed to attenuated infarct expansion.

Several laboratories have reported the presence of ventricular rupture in the C57BL/6J inbred strain during the first week after permanent coronary occlusion (Heymans et al., 1999; Askari et al., 2003; Hayashidani et al., 2003; Sun et al., 2004; Tao et al., 2004; Gao et al., 2005; Matsumura, 2005), which our results reproduced (Table 1). Rupture is an acute complication of MI in at least two other common inbred strains (Gao et al., 2005), and its rate is strain- and gender-dependent. However, the strain $(\mathrm{FVB} / \mathrm{N})$ with a rupture rate lower than the C57 is quite susceptible to acute heart failure; $45 \%$ of males succumb to this complication in the first 7 days post-MI (Gao et al., 2005). Based on these results, the total acute death rate of $8 \%$ and the rupture rate of $0 \%$ in male MRL mice reported here is remarkable.

The C57 transcriptome has been studied 1 day after permanent coronary occlusion (Harpster et al., 2006), in addition to earlier and later time points. A comparison of upregulated acute inflammation transcripts with our data revealed that $66 \%$ of the 88 transcripts common to the datasets were significantly expressed $(P<0.05)$ in both, and $100 \%$ of those were upregulated, suggesting strong similarities between the studies. The MRL transcriptome has not been studied at 1 or 5 days post-MI.

Several lines of evidence support a role for leukocyte infiltration in precipitating rupture post-MI (Heymans et al., 1999; Sun et al., 2004; Gao et al., 2005). Depletion of leukocytes has been shown to protect against rupture (Heymans et al., 1999). Moreover, the neutrophils likely to compose the majority of the CD $45^{+}$cells on day 2 post-MI (Heymans et al., 1999; Yang et al., 2002; Tao et al., 2004) are thought to be the source of matrix metalloproteinase (MMP)-9 (Lindsey et al., 2001; Tao et al., 2004; Chen et al., 2005), which may have a prominent role in degrading the existing ECM and precipitating rupture (Heymans et al., 1999; Tao et al., 2004). Gelatin zymography for MMP-9 in all three strains revealed a slight trend toward reduced gelatinolytic activity in the MRL LV and RV compared to the C57 and LG/J LV and RV, but the trend did not reach statistical significance. Expression of the inflammatory cytokines TNF- $\alpha$ and IL- $1 \beta$ was not different between the strains, but the potent neutrophil chemoattractant Cxcl7 (Walz et al., 1989) was strongly induced (48-fold) in the C57 day 1 infarct and not in the MRL. It is currently unknown whether apoptosis contributes to the likelihood of rupture after MI in mice, but a reduction in the number of TUNEL-positive myocytes in the acute phase post-MI using erythropoietin (EPO) treatment has been associated with significantly increased acute survival (Brunner et al., 2009).

Together, these studies and our data suggest that reduced apoptosis and leukocyte infiltration could contribute to the increased survival of MRL mice by providing some protection against rupture.

Our data also showed that MRL hearts healed chronically with smaller scars and attenuated remodeling compared with C57 hearts after MI. Several studies have linked attenuated acute apoptosis (Suzuki et al., 2001; Moon et al., 2003; Roubille et al., 2007; Yao et al., 2007;

Brunner et al., 2009) and inflammation (Erlich et al., 2000; Yao et al., 2007; Liehn et al., 2008) with reduced infarct scar size after MI, suggesting that the reductions we observed in TUNEL-positive nuclei and CD45 cell infiltration early after MI could potentially contribute to improved chronic healing in the MRL.

A number of other groups have studied post-MI healing in MRL mice and should be mentioned (Oh et al., 2004; Abdullah et al., 2005; Naseem et al., 2007; Cimini et al., 2008; Grisel et al., 2008; Robey \& Murry, 2008). Our finding of increased acute survival in the MRL confirms and extends that of Naseem and colleagues (2007), who reported a drop in 
C57 survival after 1 week. However, improved chronic healing had not yet been observed. Two of these groups used male MRL mice and permanent coronary occlusion (Naseem $e t$ al., 2007; Robey \& Murry, 2008), similar to the present study. One reported a survival rate of 90\% in C57 mice (Robey \& Murry, 2008), which is inconsistent with other studies (Heymans et al., 1999; Askari et al., 2003; Cavasin et al., 2003; Hayashidani et al., 2003; Sun et al., 2004; Tao et al., 2004; Gao et al., 2005; Matsumura, 2005), and may be a consequence of vendor (Harlan vs. Jackson Laboratories). Naseem et al. (2007) reported a decrease in LV dilation in MRL mice, consistent with the attenuated infarct expansion index we observed in the MRL, but no difference in 30-day infarct scar size between C57 and MRL hearts. The present study may have had more power to detect a difference in infarct scar size with group sizes of $10-11$, compared to groups of 3 in Naseem et al. (2007).

\section{Limitations}

Because we did not measure collateral flow, we cannot rule out the possibility of its contribution to the differences we observed in survival and healing between strains. However, post-infarct collateral flow as a fraction of non-ischemic flow has been measured at just 6\% in the rat, compared with nearly 16\% in the dog (Maxwell et al., 1987); neither rats nor mice have a vessel comparable to the circumflex artery found in larger mammals (Johns \& Olson, 1954). We believe it is probable that the presence of a physiologically significant difference in collateral flow between strains would lead to differences in initial infarct size, infarct/risk area, or 5-day infarct size, which we did not observe in the present study. However, a limitation of the initial infarct size measurement by TTC is the low sample number in the LG/J group $(n=3)$, and we cannot robustly exclude the possibility that initial infarct size was different in this strain than the others.

Concerning the hemodynamic data, the heart rates we report here are lower than the expected rate of $450-600 \mathrm{bpm}$. The anesthesia doses required by our Institutional Animal Care guidelines probably resulted in a deeper anesthesia plane than is common for hemodynamic measurements. With $1-\beta$ roughly equal to 0.6 , this part of the study was also somewhat under-powered. With the variability present in the heart rates of C57 and MRL mice, we cannot exclude the possibility that they may have been different if the sample size had been larger than 6 in each group.

The ex vivo measurements of infarct thickness reported here are not ideal because the hearts were not fixed at a physiological pressure. Owing to the potential for infarct tissue to deform under pressure, a more accurate representation of infarct thickness would be an in vivo measurement using a non-invasive method such as echocardiography.

Another limitation of the present study is that we did not show a direct causal relationship between the acute reductions in apoptosis and $\mathrm{CD} 45^{+}$cell infiltration and increased survival or improved healing. Additionally, we cannot rule out the possibility that in MRL hearts these acute parameters reached levels equivalent to those in C57 hearts before 1 day or beyond 5 days post-MI, and thus did not have an effect on differential survival or chronic healing. However, we believe that the previous literature in this area, as discussed above, provides support for the conclusions presented in the current study.

\section{Conclusions}

The results presented here support the idea of the MRL mouse as an intriguing, though complex, model of improved post-MI survival and healing. Our data suggest that these phenotypes could be derived in part from reductions in apoptosis and inflammatory infiltration. The intermediate survival, apoptosis, and inflammation characteristics of the parental LG/J strain indicate that genetic background may contribute significantly to MRL

Exp Physiol. Author manuscript; available in PMC 2013 January 1. 
traits, but also imply that the greater attenuation of apoptosis and inflammation in the MRL heart post-MI may be related to the increased survival displayed by this strain. Finally, these data support placing a focus on attenuating both apoptosis and inflammation in future studies designed to augment post-MI cardioprotection in the mammalian heart.

\section{Supplementary Material}

Refer to Web version on PubMed Central for supplementary material.

\section{Acknowledgments}

The authors wish to thank Angie Li, Amie Forsber, James Kwok, Saleh Amirriazi and Jun Seob Shin for their technical assistance; the UCSD Histology Core for pathology consultation and specialized staining procedures; and Berlex Biosciences for providing the Affymetrix microarrays. Author current addresses: D.L.H., W.L. Gore \& Associates, Flagstaff, AZ; P.H.C., School of Engineering and Applied Sciences, Harvard University, Cambridge, MA; H.-C.K., Department of Clinical Science, Abbott Vascular, Santa Clara, CA; K.D.Y., Knowledge Synthesis, Berkeley, CA.

Grants

This work was supported by NIH/NHLBI 5P01HL46345-12 (Kirk U. Knowlton, A.D.M); the National Biomedical Computational Resource, NIH/NCRR P41RR08605-16 (Peter W. Arzberger, A.D.M); NIH IRACDA GM068524 (A.C.Z); NIH RO1 HL070867 and NIH RO1 HL074066 (S.M.E).

\section{References}

The Jackson Laboratories JAXR Mice Data Sheet, Stock No. 000486, vol. 2006.

Abdullah I, Lepore JJ, Epstein JA, Parmacek MS, Gruber PJ. MRL mice fail to heal the heart in response to ischemia-reperfusion injury. Wound Rep Regen. 2005; 13:205-208.

Ashton, KJ.; Headrick, JP. Quantitative (Real-Time) RT-PCR in Cardiovascular Research. In: Zhang, J.; Rokosh, G., editors. Cardiac Gene Expression: Methods and Protocols. Vol. 366. Humana Press; Totowa, NJ: 2007. p. 121-143.

Askari AT, Brennan M-L, Zhou X, Drinko J, Morehead A, Thomas JD, Topol EJ, Hazen SL, Penn MS. Myeloperoxidase and Plasminogen Activator Inhibitor 1 Play a Central Role in Ventricular Remodeling after Myocardial Infarction. J Exp Med. 2003; 197:615-624. [PubMed: 12615902]

Au PYB, Martin N, Chau H, Moemeni B, Chia M, Liu F-F, Minden M, Yeh W-C. The oncogene PDGF-B provides a key switch from cell death to survival induced by TNF. Oncogene. 2005; 24:3196-3205. [PubMed: 15735680]

Blankenhorn E, Bryan G, Kossenkov A, Clark L, Zhang X-M, Chang C, Horng W, Pletscher L, Cheverud J, Showe L, Heber-Katz E. Genetic loci that regulate healing and regeneration in LG/J and SM/J mice. Mamm Genome. 2009; 20:720-733. [PubMed: 19760323]

Boyle MP, Weisman HF. Limitation of infarct expansion and ventricular remodeling by late reperfusion. Study of time course and mechanism in a rat model. Circulation. 1993; 88:2872-2883. [PubMed: 8252700]

Brunner S, Winogradow J, Huber BC, Zaruba M-M, Fischer R, David R, Assmann G, Herbach N, Wanke R, Mueller-Hoecker J, Franz W-M. Erythropoietin administration after myocardial infarction in mice attenuates ischemic cardiomyopathy associated with enhanced homing of bone marrow-derived progenitor cells via the CXCR-4/SDF-1 axis. FASEB J. 2009; 23:351-361. [PubMed: 18827024]

Campbell P, Hunt D, Jones Y, Harwood F, Omens JH, McCulloch AD. Effects of Biglycan Deficiency on Myocardial Infarct Structure and Mechanics. Mol Cell Biomech. 2008; 5:27-35. [PubMed: 18524244]

Cavasin MA, Sankey SS, Yu A-L, Menon S, Yang X-P. Estrogen and testosterone have opposing effects on chronic cardiac remodeling and function in mice with myocardial infarction. Am J Physiol Heart Circ Physiol. 2003; 284:H1560-1569. [PubMed: 12560213] 
Chen J, Tung C-H, Allport JR, Chen S, Weissleder R, Huang PL. Near-Infrared Fluorescent Imaging of Matrix Metalloproteinase Activity After Myocardial Infarction. Circulation. 2005; 111:18001805. [PubMed: 15809374]

Cimini M, Fazel S, Fujii H, Zhou S, Tang G, Weisel RD, Li R-K. The MRL mouse heart does not recover ventricular function after a myocardial infarction. Cardiovasc Pathol. 2008; 17:32-39. [PubMed: 18160058]

Clark LD, Clark RK, Heber-Katz E. A New Murine Model for Mammalian Wound Repair and Regeneration. Clin Immunol Immunopathol. 1998; 88:35-45. [PubMed: 9683548]

Doniger S, Salomonis N, Dahlquist K, Vranizan K, Lawlor S, Conklin B. MAPPFinder: using Gene Ontology and GenMAPP to create a global gene-expression profile from microarray data. Genome Biol. 2003; 4:R7. [PubMed: 12540299]

Erlich JH, Boyle EM, Labriola J, Kovacich JC, Santucci RA, Fearns C, Morgan EN, Yun W, Luther T, Kojikawa O, Martin TR, Pohlman TH, Verrier ED, Mackman N. Inhibition of the Tissue FactorThrombin Pathway Limits Infarct Size after Myocardial Ischemia-Reperfusion Injury by Reducing Inflammation. Am J Pathol. 2000; 157:1849-1862. [PubMed: 11106558]

Gao X-M, Xu Q, Kiriazis H, Dart AM, Du X-J. Mouse model of post-infarct ventricular rupture: time course, strain- and gender-dependency, tensile strength, and histopathology. Cardiovasc Res. 2005; 65:469-477. [PubMed: 15639486]

Gentleman R, Carey V, Bates D, Bolstad B, Dettling M, Dudoit S, Ellis B, Gautier L, Ge Y, Gentry J, Hornik K, Hothorn T, Huber W, Iacus S, Irizarry R, Leisch F, Li C, Maechler M, Rossini A, Sawitzki G, Smith C, Smyth G, Tierney L, Yang J, Zhang J. Bioconductor: open software development for computational biology and bioinformatics. Genome Biol. 2004; 5:R80. [PubMed: 15461798]

Grisel P, Meinhardt A, Lehr H-A, Kappenberger L, Barrandon Y, Vassalli G. The MRL mouse repairs both cryogenic and ischemic myocardial infarcts with scar. Cardiovasc Pathol. 2008; 17:14-22. [PubMed: 18160056]

Harpster M, Bandyopadhyay S, Thomas D, Ivanov P, Keele J, Pineguina N, Gao B, Amarendran V, Gomelsky M, McCormick R, Stayton M. Earliest changes in the left ventricular transcriptome post-myocardial infarction. Mamm Genome. 2006; 17:701-715. [PubMed: 16845475]

Hayashidani S, Tsutsui H, Ikeuchi M, Shiomi T, Matsusaka H, Kubota T, Imanaka-Yoshida K, Itoh T, Takeshita A. Targeted deletion of MMP-2 attenuates early LV rupture and late remodeling after experimental myocardial infarction. Am J Physiol Heart Circ Physiol. 2003; 285:H1229-1235. [PubMed: 12775562]

Heymans S, Luttun A, Nuyens D, Theilmeier G, Creemers E, Moons L, Dyspersin GD, Cleutjens JPM, Shipley M, Angellilo A, Levi M, Nu[beta]e O, Baker A, Keshet E, Lupu F, Herbert J-M, Smits JFM, Shapiro SD, Baes M, Borgers M, Collen D, Daemen MJAP, Carmeliet P. Inhibition of plasminogen activators or matrix metalloproteinases prevents cardiac rupture but impairs therapeutic angiogenesis and causes cardiac failure. Nat Med. 1999; 5:1135-1142. [PubMed: 10502816]

Hutchins G, Bulkley B. Infarct expansion versus extension: two different complications of acute myocardial infarction. Am J Cardiol. 1978; 41:1127-1132. [PubMed: 665522]

Johns TNP, Olson BJ. Experimental Myocardial Infarction: A Method of Coronary Occlusion in Small Animals. Ann Surg. 1954; 140:675-682. [PubMed: 13208115]

Li X, Gu W, Masinde G, Hamilton-Ulland M, Xu S, Mohan S, Baylink DJ. Genetic control of the rate of wound healing in mice. Heredity. 2001; 86:668-674. [PubMed: 11595047]

Liehn EA, Merx MW, Postea O, Becher S, Djalali-Talab Y, Shagdarsuren E, Kelm M, Zernecke A, Weber C. Ccr1 deficiency reduces inflammatory remodelling and preserves left ventricular function after myocardial infarction. J Cell Mol Med. 2008; 12:496-506. [PubMed: 18088392]

Lindsey M, Wedin K, Brown MD, Keller C, Evans AJ, Smolen J, Burns AR, Rossen RD, Michael L, Entman M. Matrix-Dependent Mechanism of Neutrophil-Mediated Release and Activation of Matrix Metalloproteinase 9 in Myocardial Ischemia/Reperfusion. Circulation. 2001; 103:21812187. [PubMed: 11331260]

Matsumura, S-i. Targeted deletion or pharmacological inhibition of MMP-2 prevents cardiac rupture after myocardial infarction in mice. J Clin Invest. 2005; 115:599-609. [PubMed: 15711638] 
Maxwell MP, Hearse DJ, Yellon DM. Species variation in the coronary collateral circulation during regional myocardial ischaemia: a critical determinant of the rate of evolution and extent of myocardial infarction. Cardiovasc Res. 1987; 21:737-746. [PubMed: 3440266]

Moon C, Krawczyk M, Ahn D, Ahmet I, Paik D, Lakatta EG, Talan MI. Erythropoietin reduces myocardial infarction and left ventricular functional decline after coronary artery ligation in rats. PNAS. 2003; 100:11612-11617. [PubMed: 14500913]

Naseem RH, Meeson AP, Michael DiMaio J, White MD, Kallhoff J, Humphries C, Goetsch SC, De Windt LJ, Williams MA, Garry MG, Garry DJ. Reparative myocardial mechanisms in adult C57BL/6 and MRL mice following injury. Physiol Genomics. 2007; 30:44-52. [PubMed: 17327495]

Oh Y-S, Thomson LEJ, Fishbein MC, Berman DS, Sharifi B, Chen P-S. Scar formation after ischemic myocardial injury in MRL mice. Cardiovasc Pathol. 2004; 13:203-206. [PubMed: 15210135]

Pattanapanyasat K, Pengruangrojanachai V, Thepthai C, Suwanagool S, Wasi C. Flow cytometric three-color determination of CD4 T-lymphocytes on blood specimens from AIDS patients who have a large number of contaminating non-lymphocytes. Asian Pac J Allergy Immunol. 1994; 12:105-109. [PubMed: 7612102]

Pfeffer JM, Pfeffer MA, Fletcher PJ, Braunwald E. Progressive ventricular remodeling in rat with myocardial infarction. Am J Physiol Heart Circ Physiol. 1991; 260:H1406-1414.

Pfeffer MA, Braunwald E. Ventricular Remodeling After Myocardial Infarction. Circulation. 1990; 81:1161-1172. [PubMed: 2138525]

Pfeffer MA, Pfeffer JM, Fishbein MC, Fletcher PJ, Spadaro J, Kloner RA, Braunwald E. Myocardial infarct size and ventricular function in rats. Circ Res. 1979; 44:503-512. [PubMed: 428047]

Reffelmann T, Hale SL, Dow JS, Kloner RA. No-Reflow Phenomenon Persists Long-Term After Ischemia/Reperfusion in the Rat and Predicts Infarct Expansion. Circulation. 2003; 108:29112917. [PubMed: 14656914]

Robey TE, Murry CE. Absence of regeneration in the MRL/MpJ mouse heart following infarction or cryoinjury. Cardiovasc Pathol. 2008; 17:6-13. [PubMed: 18160055]

Roubille F, Combes S, Leal-Sanchez J, Barrere, Christian, Cransac F, Sportouch-Dukhan C, Gahide G, Serre I, Kupfer E, Richard S, Hueber A-O, Nargeot J, Piot C, Barrere-Lemaire S. Myocardial Expression of a Dominant-Negative Form of Daxx Decreases Infarct Size and Attenuates Apoptosis in an In Vivo Mouse Model of Ischemia/Reperfusion Injury. Circulation. 2007; 116:2709-2717. [PubMed: 18025529]

Smyth GK. Linear models and empirical bayes methods for assessing differential expression in microarray experiments. Stat Appl Genet Mol Biol. 2004; 3:Article 3.

Sun M, Dawood F, Wen W-H, Chen M, Dixon I, Kirshenbaum LA, Liu PP. Excessive Tumor Necrosis Factor Activation After Infarction Contributes to Susceptibility of Myocardial Rupture and Left Ventricular Dysfunction. Circulation. 2004; 110:3221-3228. [PubMed: 15533863]

Suzuki K, Murtuza B, Smolenski RT, Sammut IA, Suzuki N, Kaneda Y, Yacoub MH. Overexpression of Interleukin-1 Receptor Antagonist Provides Cardioprotection Against Ischemia-Reperfusion Injury Associated With Reduction in Apoptosis. Circulation. 2001; 104:I-308-313. [PubMed: 11568074]

Tao Z-Y, Cavasin MA, Yang F, Liu Y-H, Yang X-P. Temporal changes in matrix metalloproteinase expression and inflammatory response associated with cardiac rupture after myocardial infarction in mice. Life Sci. 2004; 74:1561-1572. [PubMed: 14729404]

Ugur O, Kathryn AG, Kimberlee D-H, Edward M, William BS. NG2 proteoglycan is expressed exclusively by mural cells during vascular morphogenesis. Dev Dyn. 2001; 222:218-227. [PubMed: 11668599]

van den Borne SWM, Cleutjens JPM, Hanemaaijer R, Creemers EE, Smits JFM, Daemen MJAP, Blankesteijn WM. Increased matrix metalloproteinase- 8 and -9 activity in patients with infarct rupture after myocardial infarction. Cardiovasc Pathol. 2009; 18:37-43. [PubMed: 18402833]

van der Laan, M.; Pollard, K. Technical Report No. 93. Division of Biostatistics, Univ. of California; Berkeley: 2001. A New Algorithm for Hybrid Hierarchical Clustering with Visualization and the Bootstrap. 
Walz A, Dewald B, von Tscharner V, Baggiolini M. Effects of the neutrophil-activating peptide NAP-2, platelet basic protein, connective tissue-activating peptide III and platelet factor 4 on human neutrophils. J Exp Med. 1989; 170:1745-1750. [PubMed: 2681518]

Wu Z, Irizarry RA. Preprocessing of oligonucleotide array data. Nat Biotech. 2004; 22:656-658.

Yang F, Liu Y-H, Yang X-P, Xu J, Kapke A, Carretero OA. Myocardial Infarction and Cardiac Remodelling in Mice. Exp Physiol. 2002; 87:547-555. [PubMed: 12481929]

Yang X, Khosravi-Far R, Chang HY, Baltimore D. Daxx, a Novel Fas-Binding Protein That Activates JNK and Apoptosis. Cell. 1997; 89:1067-1076. [PubMed: 9215629]

Yao Y-Y, Yin H, Shen B, Chao L, Chao J. Tissue kallikrein infusion prevents cardiomyocyte apoptosis, inflammation and ventricular remodeling after myocardial infarction. Regul Pept. 2007; 140:12-20. [PubMed: 17196272] 


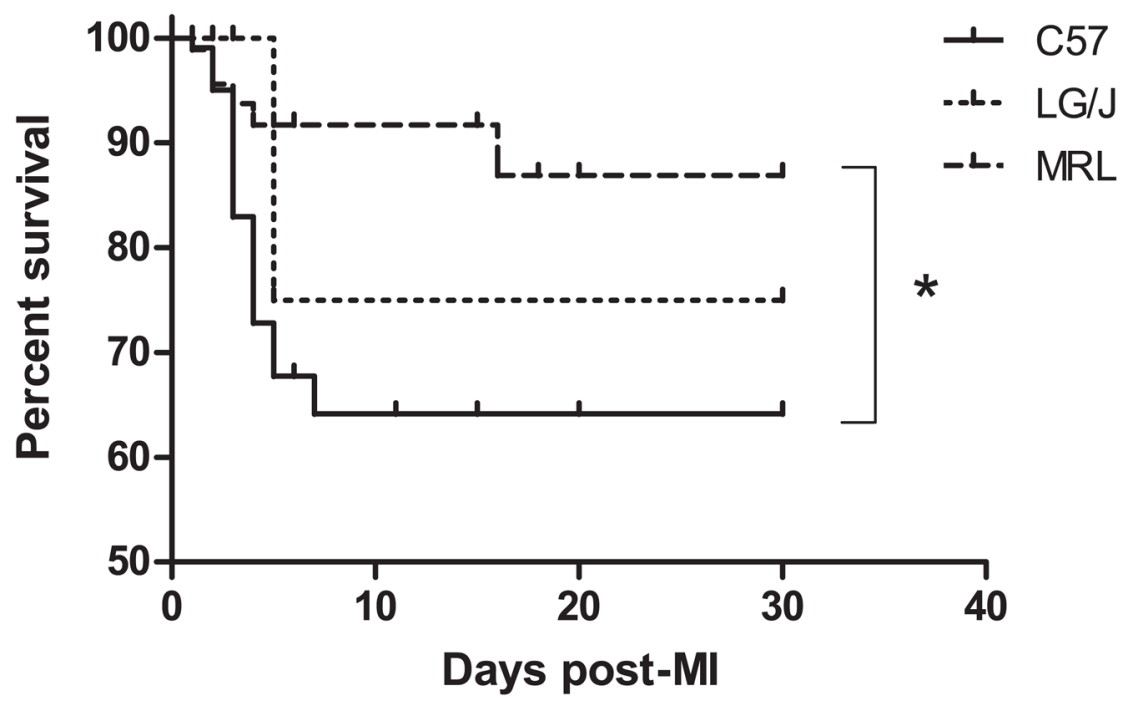

Figure 1.

Kaplan-Meier survival analysis of all 3 strains through 30 days post-MI. There was a significant trend in survival by strain $(P<0.01)$, and MRL mice $(n=90)$ had a survival advantage compared with C57 mice $(n=106 ; P<0.05)$. LG/J survival $(n=31)$ was intermediate $(P=\mathrm{NS})$. ${ }^{*}, P<0.05$. 
A

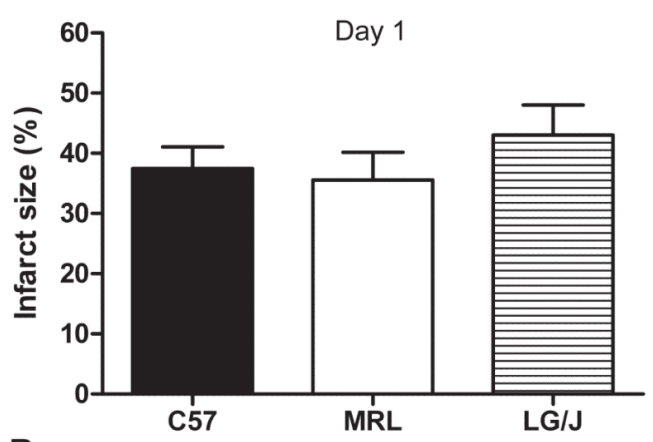

B

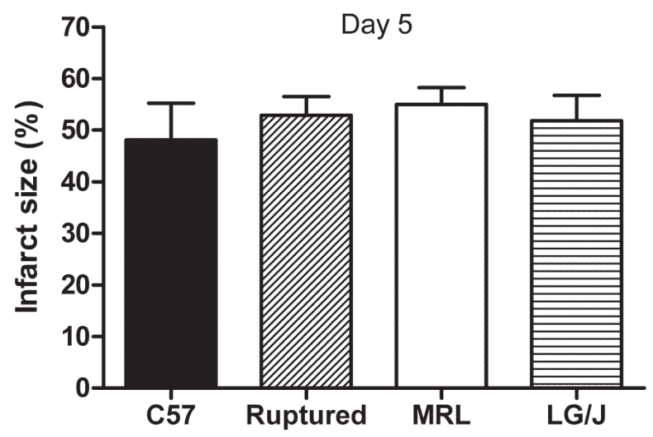

C

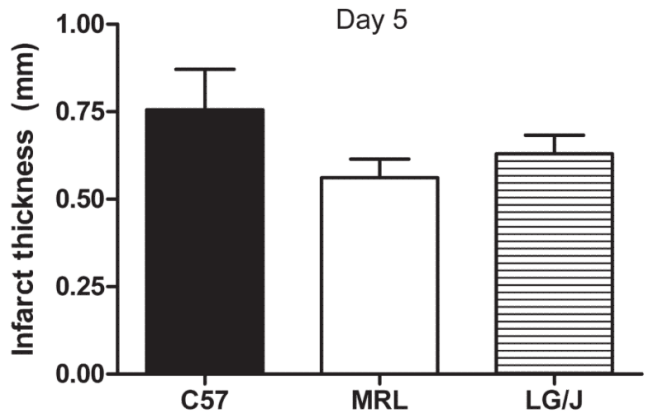

Figure 2.

(A) There was no statistically significant difference in initial infarct size 1 day post-MI between the 3 strains (C57 $n=10$, MRL $n=9$, LG/J $n=3 ; P=0.67$.). (B) There were no significant differences in infarct size after 5 days between hearts of animals sacrificed on that day and C57 hearts that ruptured on or before 5 days post-MI (C57 $n=5$, C57 ruptured hearts $n=3$, MRL $n=4, \mathrm{LG} / \mathrm{J} n=4 ; P=0.86$.). Infarct thickness was not preserved in MRL hearts 5 days post-MI $(\mathrm{C})$; there were no significant differences in infarct thickness between the strains (C57 $n=5$, MRL $n=3, \mathrm{LG} / \mathrm{J} n=4 ; P=0.32$.). 


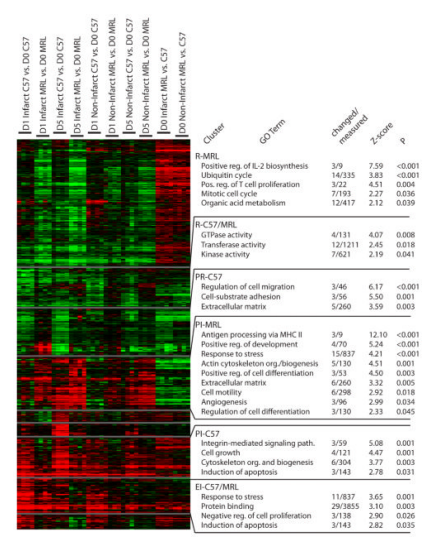

Figure 3.

HOPACH clustering and MAPPFinder annotation results. Green, downregulation; red, upregulation. Changed/measured, number of genes in cluster/number on array. Z-score explained in Doninger, et al. Abbreviations: R, repression; PR, progressive repression; PI, progressive induction; EI, early induction. C57/MRL indicates that the expression pattern was similar in both strains. 

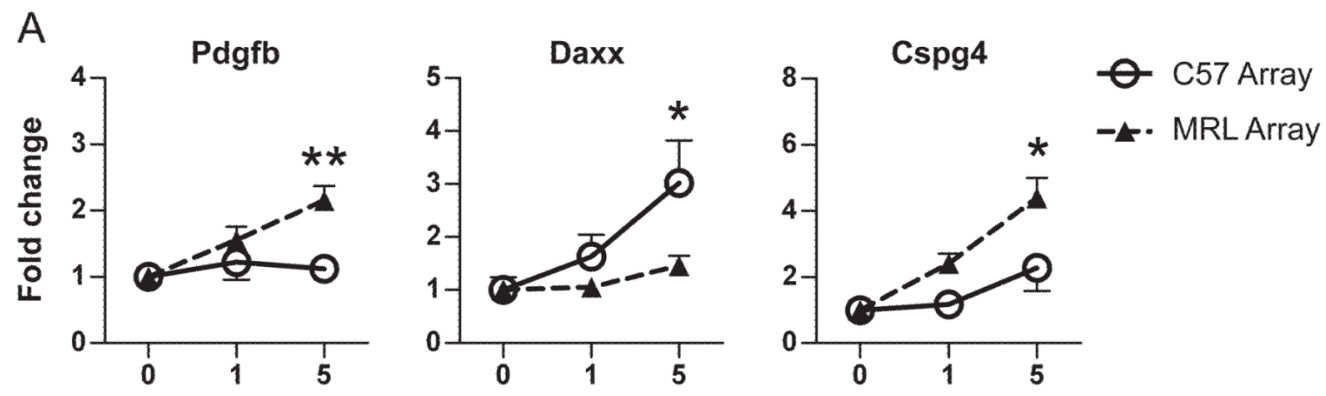

Days post-MI
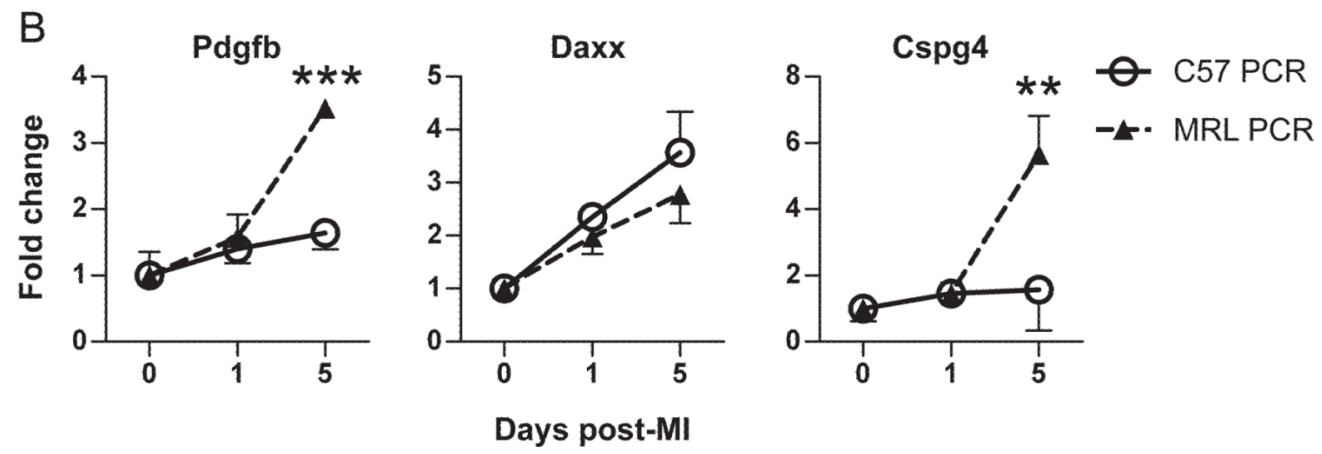

Figure 4.

Validation of Affymetrix MOE430A microarray gene expression ( $n=4$ in each group except C57 day $0 n=3$ ) before (day 0) and 1 and 5 days after MI using quantitative realtime PCR ( $n=3$ in each group). Expression patterns are similar between microarray (A) and PCR (B). Pdgfb: platelet-derived growth factor beta; Daxx: Fas death domain-associated protein; Cspg4: chondroitin sulfate proteoglycan 4 . ${ }^{*}, P<0.05$; **, $P<0.01$; ***, $P<$ 0.001. Significance was reached on day 5 only. 


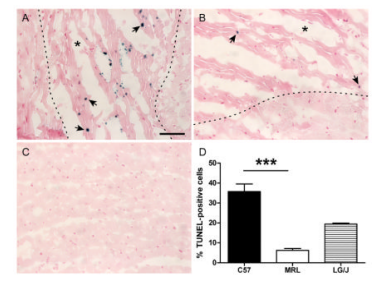

\section{Figure 5.}

TUNEL staining in the infarct border zone of C57 (A) and MRL (B) hearts 1 day post-MI ( $n$ $=6$ in each group). Arrows point to TUNEL-positive nuclei. Dotted lines denote the approximate infarct boundary. An asterisk marks the injured region. A representative image of remote LV myocardium is shown in panel C. The number of TUNEL-positive nuclei closely associated with myocytes was significantly higher in C57 hearts than MRL hearts $(P$ $=0.001)(\mathrm{D})$. There were no statistically significant differences between LG/J hearts $(n=4)$ and the other groups. Bar $=50 \mu \mathrm{m}$. ***, $P=0.001$. 


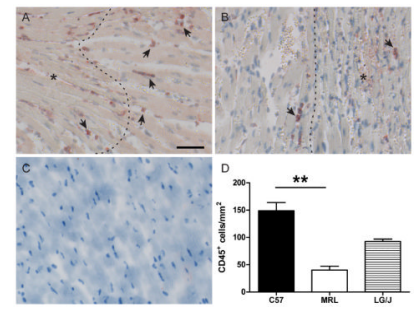

Figure 6.

CD45 ${ }^{+}$cells (arrows) in the infarct border zone of C57 (A) and MRL (B) hearts 2 days postMI (C57 $n=6$, MRL $n=5)$. Dotted lines denote the approximate infarct boundary. An asterisk marks the injured region. A representative uninjured control is shown in panel C. There were significantly more CD $45^{+}$cells $/ \mathrm{mm}^{2}$ in the C57 infarct border zone than in the MRL border zone $(P<0.01)(D)$. There were no statistically significant differences between $\mathrm{LG} / \mathrm{J}$ hearts $(n=4)$ and the other groups. Bar $=50 \mu \mathrm{m} . * *, P<0.01$. 
A

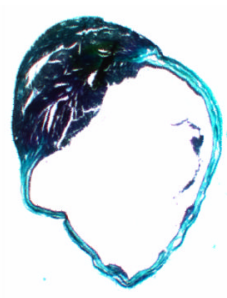

C57

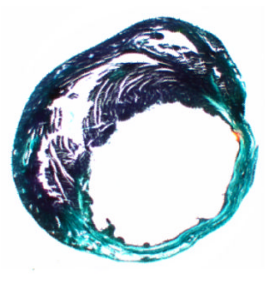

MRL
C

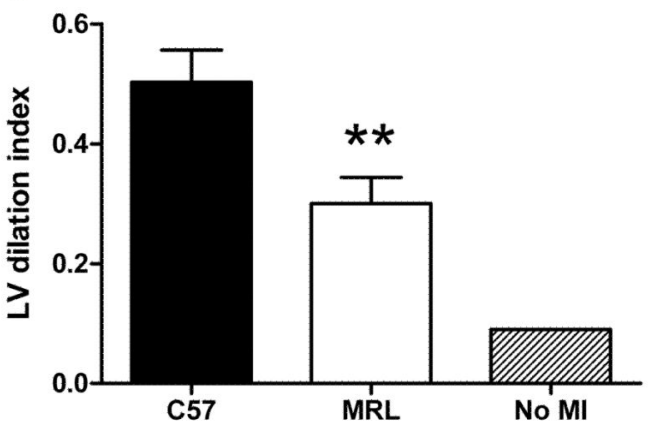

Figure 7. $P<0.05 ; * *, P<0.01$.
B

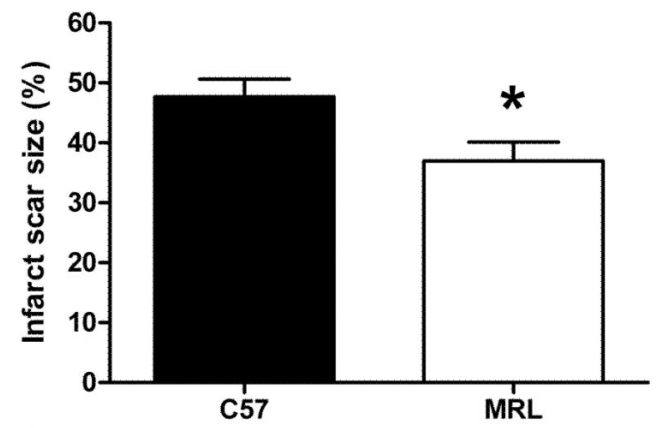

D

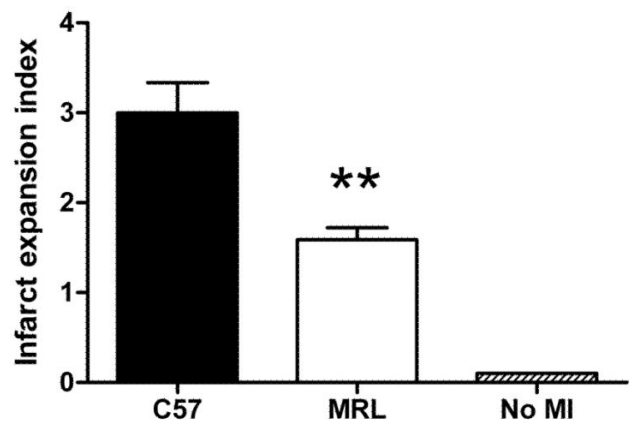

Deleterious remodeling in MRL hearts $(n=11)$ was attenuated compared with C57 hearts $(n$ = 10) 30 days post-MI. Representative trichrome-stained sections from C57 (left) and MRL (right) hearts (A). Infarct scar size after 30 days was significantly smaller in MRL hearts ( $P$ $<0.05$ ) (B). LV dilation (C) and infarct expansion (D) were also significantly attenuated. *, 


\section{Table 1}

Surgical data

\begin{tabular}{|c|c|c|c|}
\hline & \multicolumn{3}{|c|}{ Mouse Strain } \\
\hline & C57 & MRL & LG/J \\
\hline & \multicolumn{3}{|c|}{ Number of animals } \\
\hline MI operations & 136 & 121 & 60 \\
\hline Operative survival $(\%)^{*}$ & $114(84 \%)^{\dagger}$ & $117(97 \%)$ & $55(92 \%)$ \\
\hline Ventricular rupture (rupture rate ${ }^{\frac{1}{f}}$ ) & $17(29 \%)$ & $0(0 \%)^{\circ}$ & $2(25 \%)$ \\
\hline \multicolumn{4}{|c|}{ Animals that were alive at the end of the day of surgery (day 0 ). } \\
\hline \multicolumn{4}{|l|}{$P<0.01$ vs. MRL and LG/J. } \\
\hline
\end{tabular}

Exp Physiol. Author manuscript; available in PMC 2013 January 1. 
Table 2

Hemodynamic parameters

\begin{tabular}{lccc}
\hline & \multicolumn{3}{c}{ Mouse Strain } \\
\cline { 2 - 4 } Parameter & $\mathbf{C 5 7}(\boldsymbol{n}=\mathbf{6})$ & MRL $(\boldsymbol{n}=\mathbf{6})$ & LG/J $(\boldsymbol{n}=\mathbf{3})$ \\
\hline Heart rate $(\mathrm{bpm})$ & $318 \pm 41$ & $249 \pm 18$ & $326 \pm 2$ \\
LV end-systolic pressure $(\mathrm{mmHg}))$ & $86 \pm 7$ & $81 \pm 4$ & $89 \pm 8$ \\
LV end-diastolic pressure $(\mathrm{mmHg})$ & $15 \pm 3$ & $15 \pm 2$ & $4.1 \pm 0.3^{*}$ \\
Max dP/dt (mmHg/sec) & $5586 \pm 665$ & $5024 \pm 545$ & $7854 \pm 250^{\dagger}$ \\
Min dP/dt (mmHg/sec) & $-3896 \pm 322$ & $-3685 \pm 300$ & $-4851 \pm 156$ \\
\hline$*$ & & & \\
\hline
\end{tabular}

Exp Physiol. Author manuscript; available in PMC 2013 January 1. 